\title{
Bike Sharing Atlas: Visual Analysis of Bike-Sharing Networks
}

\author{
Michael Oppermann ${ }^{1}$, Torsten Möller ${ }^{2}$ and Michael Sedlmair ${ }^{3}$ \\ University of Vienna \\ ${ }^{1}$ michael@oppermann.at, ${ }^{2}$ torsten.moeller@univie.ac.at, \\ 3michael.sedlmair@univie.ac.at
}

\begin{abstract}
In this paper, we introduce an interactive visualization system, bikesharingatlas.org, that supports the explorative data analysis of more than 468 bike-sharing networks worldwide. The system leverages a multi-coordinated view approach and innovative interaction techniques can help, for instance, to expose capacity bottlenecks, commuting patterns, and other network characteristics. Our broader goal is to illustrate how visual analysis can be used for exploring distributed, heterogeneous data from smart cities. Based on our collaboration with different target users, we present usage scenarios that show the potential of our approach to understanding bike-sharing and urban commuting behaviors.
\end{abstract}

Keywords: smart city, bike-sharing, visualization, interactive visual analysis

\section{Motivation}

The majority of the world's population is living in urban areas, and this proportion continues to grow. An increase in efficiency is needed for cities to function, and sustainable infrastructures will be essential to accommodate larger numbers of people. As digitization has become an integral part of our life, massive amounts of data from a variety of sources are generated continuously in cities worldwide. Leveraging this data intelligently offers great potential towards smarter and more efficient cities.

Although having these immense datasets at our fingertips, we often lag behind in supporting people to intelligently leverage the huge amount of data that is produced daily. A city planner might want to identify and better understand commuting patterns in order to develop more robust and cohesive transportation infrastructures. A sociologist wants to study local effects of job density and residential segregation on society, or wants to perform other data-intensive tasks like cross-country comparisons of urbanization. However, currently most of the data comes in machine-readable form only and hence is hard to access for people without sophisticated computational and statistical skills.

In this paper, our goal is to illustrate how interactive visualization can help to open the data that is produced in smart cities to a wider audience. We believe that interactive visualization can help us to engage users, and to interactively explore and understand collected data from smart city sensors, in order to make life more comfortable, safer and sustainable.

Towards this goal, we take public bike-sharing systems as an example and show how visualization can help to better leverage the data produced by these systems. Bike-sharing systems have been established as permanent components in urban passenger transport since 1996 [1]. Being increasingly digitized, these systems nowadays produce data that can reveal interesting insights, not only into patterns of bicycle usage, but also underlying spatiotemporal dynamics of a city, as Froehlich et al., [2] and Wood et al., [3] pointed out.

Received (July 28, 2017), Review Result (September 21, 2017), Accepted (October 12, 2017) 
As a first step, we recorded continually, over a period of 17 months, distributed data from several hundred bike-sharing networks worldwide. We aggregated the data, combined it with other data sources and derived characteristic network metrics.

In the course of an iterative design process we built a visualization tool with a variety of viewing options to offer multiple different perspectives on the data. Various design considerations were inspired and strongly shaped by our interactions with different target user groups: operators of a bike-sharing network, urban sociology researchers, public authorities, city planners, and the general public. To illustrate the value of this visualization approach, we provide four different usage scenarios: (1) how visualizing long-term recorded data can help to better understand commuting patterns in a city, (2) how the combination with other data sources can reveal interesting insights, (3) how our approach allows to compare systems world-wide through a shared global perspective (4) and how the general public can benefit from recording and visualizing this data. While our focus is on bike-sharing networks, we believe that - with the proliferation of globalization and mega cities - such data-driven and visual approaches will become increasingly important for other aspects of open urban data as well.

In summary, our main contributions are:

- We recorded and made openly available a repository of open bike-sharing data from 380 different cities over a period of 17 months and additionally data from 88 cities over a period of 4 months.

- We designed an online interactive visualization that makes the data accessible to users with a wide range of expertise.

- We describe a set of four usage scenarios to illustrate the benefits of our approach for different target users.

\section{Related Work}

Bike-sharing data has been used in different contexts before. Here, we review this previous work with the goal to contextualize our work and provide the interested reader with further pointers into the visualization and data analysis literature.

The most prominent analyses of bike-sharing data this far have been statistical analyses. The goals of these analyses were, for instance, to help system operators to improve the location of stations [19], or re-balance bikes between stations [20]. While these approaches look for an algorithmic solution for a clearly defined task, our goal is different in that we want to make the data accessible, visible, and explorable to a wide range of potential users.

Towards this goal, we took the approach of interactive visualization, which is wellknown to support such exploratory endeavors [21, 22]. It provides an essential way to support the user in a hypothesis generation and decision-making process.

There exist some efforts that focus on the visual analysis of individual bike-sharing networks. Studying the spatial distribution of journeys and peak-time behaviors in London $[3,23]$, analyzing the system dynamics over a 10-month long period in New York City [24] or providing a tool to interactively explore the impact of demographic and weather factors on bike-share ridership in Boston [25]. In a broader context, there are several approaches that use other data sources in conjunction with visualizations to solve problems that are abstractly similar to the one of bike-sharing networks. These works use other smart city sensors, such as mobile phone data [26], public transport data [27] or social media data [26] to explore opportunities that arise from this type of data-driven analysis for smart cities. Ferreira et al., [28] used taxi trip data and Miranda et al., [29] utilized meta-data of Flickr images to visually explore and understand behavioral patterns in New York City. With these tools we share the goal to make the data accessible and to provide different levels of analysis. However, all these approaches take a local perspective on one city and are often 
limited to a specific target group, while our goal is to investigate how to expand such approaches to a more global level and for a broad range of users.

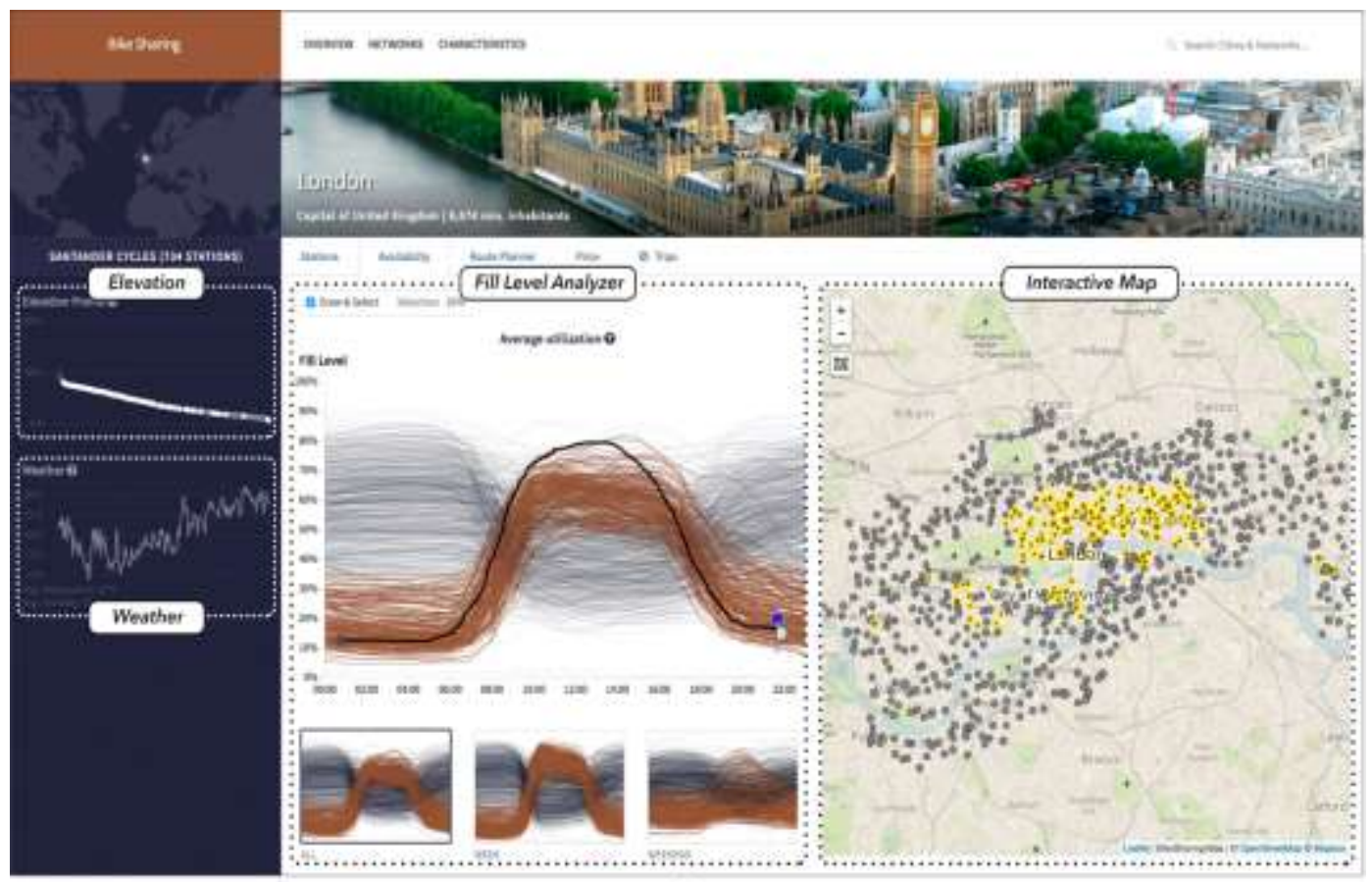

(a)

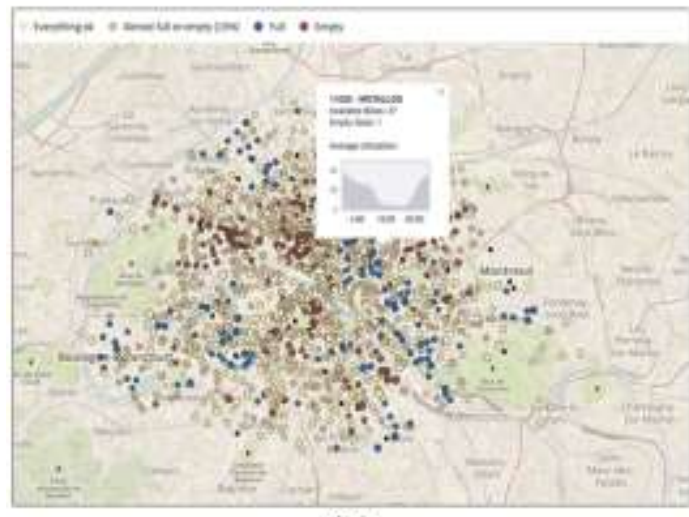

(b)

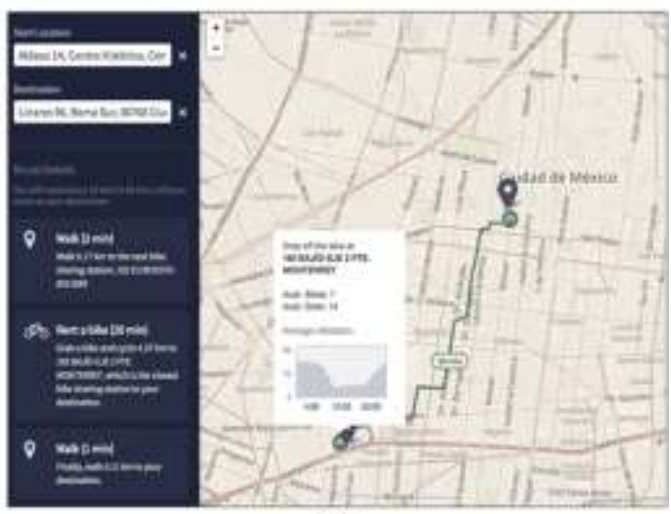

(c)

Figure 1. Screenshots of the Web Interface when Focused on a Specific City: (a) The Fill Level Analyzer View Exposes Bike-Sharing Commuting Behaviors in London, U.K. Each Line Represents an Individual Bike-Sharing Station and Shows the Daily Average Utilization. Selected Stations are Highlighted in all Coordinated Views. (b) Current Overview of Station Fill Levels in Paris, France. Tooltips Help to Predict the Availability of Bikes and Empty Docks at Certain Times during the Day. As Shown in the Legend on top of the Map, Blue Dots Indicate Full Stations, and Red Dots Empty Ones. Circle Size Encodes the Overall Capacity of a Station. (c) The Route Planner Shows the Fastest Bike-Sharing Route between Two Endpoints in Mexico City

In terms of analyzing multiple cities, Austwick et al., [30] used statistics and visualization techniques to compare five different cities. Bargar et al., [31] proposed an 
application for comparing usage patterns between different bike-sharing programs from up to three different cities and Nagel et al., [32] exhibited, in a public gallery space, several visualizations for casually analyzing three different bike-sharing networks. Most closely related to our approach are O'Brien [33], Meddin et al., [34] and the platform citybik.es [4], who all used map-based tools to show the locations of bike-sharing networks worldwide. Although these tools provide an overview of networks, they are based on very homogeneous data sources and do not take a long-term and contextual perspective, as we do. Meddin's Bike-sharing Map [34] is based on Google Maps and administered manually.

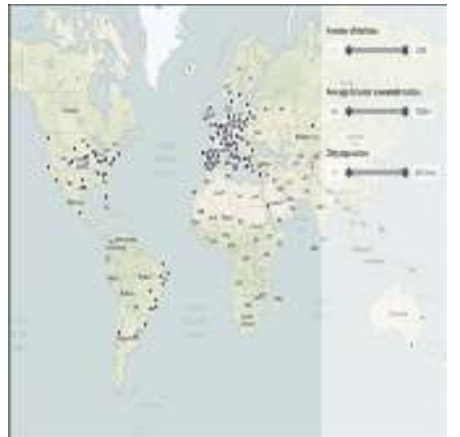

(a) Interactive Filterable Map Provides a

Geographical Overview of all Networks

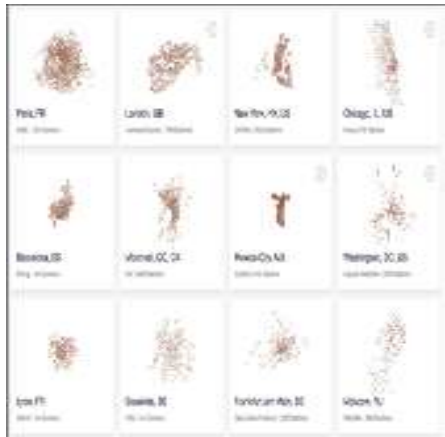

(b) Small Multiples are Good Indicators of the Size,

Structure and Density Of Networks

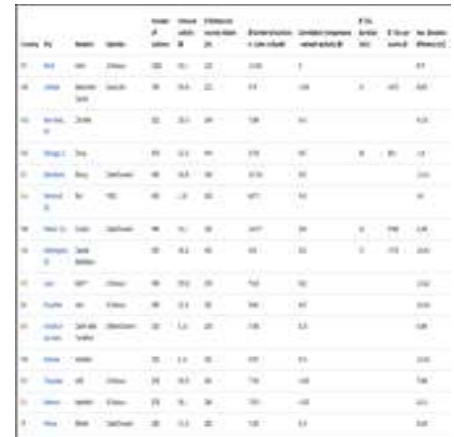

(c) Table View with Detailed Statistical Data of all Networks

Figure 2. Screenshots of Different Overview Components of the Tool

It provides a high-level overview of bike-sharing programs around the world and includes also networks that are currently built or networks, for example in Asia, that don't provide live station data. Nevertheless, the navigation and the visual encodings weaken the usability. Citybik.es [4] uses the same information base as we do but is limited to showing live station fill levels. O'Brien's Bike Share Map [33] goes one step further by showing data from the last 24 hours but also does not offer a long-term perspective and the possibility for in-depth analyses. O'Brien et al., [7] proposed a classification of 38 systems based on spatio-temporal characteristics and demonstrated thereby the opportunities of a higher-level view. In contrast to this work, which is a static analysis and a discussion of various insights, our goal was an interactive visualization tool that allows for dynamic and interactive exploration of the underlying data.

\section{Data Acquisition and Preprocessing}

A bike-sharing network, by our definition, has a certain number of stations and often thousands of bikes circulating in the network. Every station is composed of docking spaces and has therefore a finite capacity. The number of bikes in each station, we call it fill level, is highly dynamic and also a significant factor for the functioning of a system. Many operators make their collected data available to the public and contribute to various open data initiatives. A few cities share detailed historical data about completed trips but the majority provide only information about current station fill levels. In our application we put the focus on this type of information, to cover as many cities as possible.

We started collecting data from 380 networks at the beginning and added new networks continuously. Our database is now composed of data from 468 networks in 45 different countries, with more than 21,500 stations. We gathered the data through api.citybik.es [4] or directly from the websites of the bike-sharing operators. Over a period of 17 months and for 380 networks we have logged the number of available bikes and empty docks for each station in a 15 min interval. Additionally, we further recorded this information from 88 
other networks over a 4-month period. The current version supports only a few systems from Asia, due to access limitations [4] but our tool provides interfaces to easily connect additional networks in the future.

For data quality assurance purposes, we automatically verify if the geo-coordinates are plausible and lie in a specific region. We have also checked if the data from api.citybik.es is consistent with the information that is published on the websites of the respective bikesharing operators. If we observe that the fill levels of a network are not updated anymore, we disable certain features or the whole network. Due to the large number of networks and different data providers, outages and some uncertainties are inevitable. However, the aggregation of several months creates a balancing effect and counteracts these problems.

For all 468 networks, we enhance the platform with hourly weather records (temperature) and elevation profiles (altitude of each station). We get access to current weather information via OpenWeatherMap ${ }^{1}$ and the elevation profiles were loaded by means of the Google Maps Elevation API ${ }^{2}$. Additionally, we use Google Maps API, Mapbox and Open Street Map for map-related services.

In total we collected more than 830 million fill levels, that offer a large potential for various analysis and prediction tasks, while also confronting us with additional processing challenges. For this reason, we integrated multiple preprocessing steps to break down the database into smaller chunks that can be loaded during runtime. High-level network metrics are also generated in advance. In addition to the number of stations per network, their elevation profiles and the average number of docks per station, we calculated the following characteristics for filter, ranking and comparison purposes.

- Average number of stations within a $2 \mathrm{~km}$ radius: Starting from each station we counted the number of reachable stations in a $2 \mathrm{~km}$ radius and averaged across it. This radius corresponds to the mean trip distance in Vienna.

- Average nearest neighbor distance: For each station, we compute the distance to its nearest station. We then average these distances over all stations in this bike-sharing network.

- Network activity: We defined this metric by counting the number of fill level changes for every station and normalized it to the network size, as illustrated by the pseudo code below (Algorithm 1). Due to the 15 min interval in our data retrieval, there is some natural uncertainty of this straight-forward measure for larger networks [5].

- Maximum elevation difference between the highest and lowest station without consideration of possible hills and altitude changes in between.

\section{Table 1. Calculating the Network Activity for each Bike-Sharing Network}

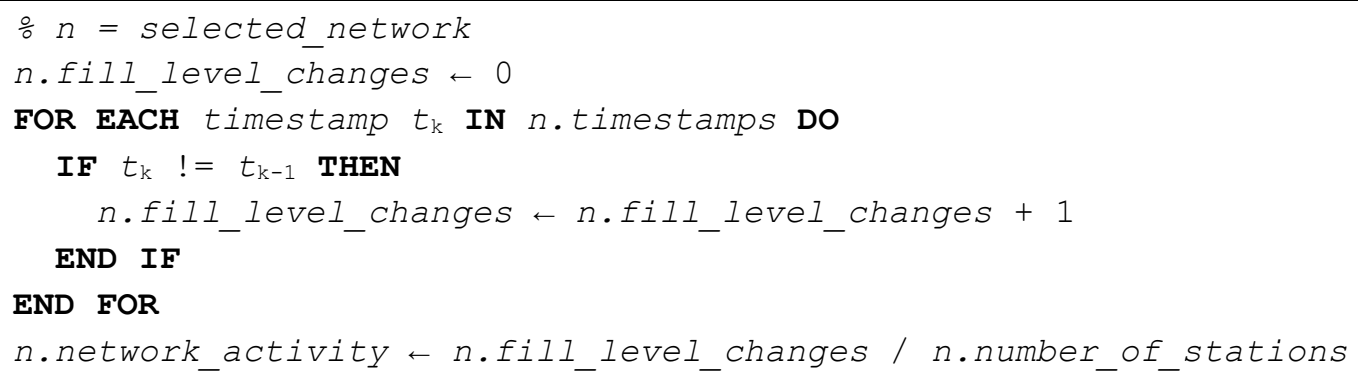

\footnotetext{
${ }^{1}$ https://openweathermap.org

${ }^{2}$ https://developers.google.com/maps/documentation/elevation/
} 


\section{Bike Sharing Atlas}

We have developed an interactive visualization system (bikesharingatlas.org) to help users with a wide range of expertise to understand and intelligently leverage data that is produced by public bike-sharing systems worldwide. Figure 1-a shows a screenshot of the web tool and a video in the supplemental material provides further details on user interactions.

In the following, we give an overview of the system and subsequently we present four selected usage scenarios. In this context, we also describe in detail primary visual and interaction design choices we made during the implementation process.

\subsection{System Overview}

Global view: Initially, the homepage of the web-platform presents an interactive map showing the geographical locations of all bike-sharing networks. To ease getting started, we also display example cities including the city of the user's current location, and those that are particularly interesting because of the available data. Two separate pages provide further high-level overviews of all networks, as shown in Figure 2 and 6. A small multiples view (Figure 2-b), for instance, provides a first impression of the size and density of the networks by visualizing them as individual vector maps. Sort functions, range sliders, and histograms allow users to explore and compare hundreds of networks in an interactive way.

Local view: The user can then select a network of a particular city and go one level deeper revealing detailed information about this network (and city). A tab navigation and multiple views provide different perspectives on the selected network and support the user in the exploration process. The currently available detail views include: (1) Interactive map with current fill levels of the network, as shown in Figure 1-b; (2) Route planner, Figure 1-c, and in Figure 1-a: (3) Fill level analyzer with historical data; (4) Time series chart with the network activity and the superimposed temperature profile; (5) Other information about the network and the city, such as bike-sharing pricing, detailed information on trips (if available), or additional weather and elevation data.

Multiple entry points, a search function, and a clear navigation structure provide an easy way to get from a global to a local view and vice versa. Moreover, we followed the idea of suggested interactivity [6], in the form of little preview videos and tooltips, to guide firsttime users through the visualization system.

\subsection{Usage Scenarios}

Over the course of the last two years, we evaluated and discussed our approach with the following target groups: Operators of a medium-sized bike-sharing network, city planners, public authorities, researchers in urban sociology, and the general public. Overall, we interviewed 15 participants in 13 single or group sessions. The insights we gained together with these people informed many design considerations and shaped the final implementation. The usage scenarios below are based on them and are meant to illustrate the potential of our interactive visualization tool.

4.2.1. Usage Scenario - Commuting Patterns: By recording and aggregating station fill levels over a period of 17 months we get an accurate picture of the daily average utilization of each station in a network. The fill level analyzer, shown in Figure 1-a, with its multiple coordinated views is based on this data and provides a new approach to identify capacity bottlenecks and commuting behaviors in bike-sharing systems. The multi-series line chart is the core element and shows, for a selected city, the average fill levels (y-axis) during the day (x-axis). Each line represents a station. Due to the different numbers of docking spaces per station, the fill levels are normalized. This contrasts from the work from O'Brien et al., [7] where all station fill levels are aggregated to get a single line. In that case, during the 
averaging process important information gets lost and it is impossible to expose critical stations that are mostly full or empty. In our proposed system, all stations are separated and the user can switch between different modes to explore, for example, fill levels only for weekends or weekdays. Additionally, there are two other perspectives: an elevation profile of all stations and an interactive map for providing the geographic context.

Dynamic linking and brushing [8], an interactive visualization technique that connects multiple views, leads to a holistic understanding of the city dynamics. As shown in Figure 1 , the user can draw a line - a hypothetical profile — on top of the multi-series line chart and our algorithm automatically selects similar stations. At the end of the drawn line a slider is displayed and allows the user to specify how many lines she wants to select (default is $20 \%$ of the lines). The selected stations are not only highlighted in the line chart (brushing), but also in all other views (linking). Linking and brushing is a well-studied technique in visualization research [37] and many systems have successfully employed it in the past. Its biggest benefit is that the users can interactively and easily cast complex queries to the data. The query is casted in a direct manipulation manner [38], that is, the user can stay in her mental model without the need to leave her current train of thought and interaction mode. The highlighting is propagated to various other views where aspects related to the user's selection visually pop out without the necessity to actively process them [39]. This approach allows the user to quickly analyze complex patterns that are distributed over multiple views. The supplemental video illustrates our linking and brushing approach.

Besides bike-sharing operators, that can use the tool to analyze capacity bottlenecks, such as frequent outages, city planners and public authorities can use the visualization system also to identify and communicate urban commuting behaviors. For example, stations in the city center of London get full during the day and empty through the night, as shown in Figure 1-a. This phenomenon is observable in many cities worldwide as illustrated in Figure 3. Milan and New York City show very similar behaviors. People from the outskirts are commuting to downtown areas, such as Fifth Avenue in New York or Piazza Duomo in Milan, in the morning and return in the evening. Mexico City's pattern is a bit more faceted and shows multiple geographical hotspots. Although our two city planner interviewees have hypothesized a considerable separation of residential and commercial spaces in certain cities, our tool exposed visible evidence for this behavior.

Barcelona and other Spanish cities show this characteristic morning commute curve too but in the afternoon it becomes vague. We can imagine that this effect might stem from the different working patterns in Spain with a longer lunch break and longer working hours in the evening [9]. This assumption could be further investigated by sociology researchers with the aid of our visualization system.

Other cities, such as Marseille or Vienna, are instead characterized by a mixed-use development, without such a clear commuting pattern in filling levels (i.e., mostly balanced fill levels).

4.2.2. Usage Scenario - Combining with Additional Data: Multiple guided brainstorming sessions with a bike-sharing operator revealed that elevation differences between stations are essential factors for the cost-efficient functioning of a system. Stations at higher altitudes tend to be empty more frequently because users are usually more downhilloriented. The bikes must be re-balanced manually by the operator. By combining the historical bike-sharing records with elevation profiles, the implemented visualization system also supports a closer investigation of this question. As part of the fill level analyzer, described in the previous usage scenario, we have integrated an elevation profile of all stations, which is also connected via linking and brushing to all other views. Instead, of a $\mathrm{u}$-shaped commuting pattern such as in Figure 1-a, average filling levels in Vienna, for example, remain mostly constant throughout the day but show clear evidence of fewer available bikes at higher altitudes (see Figure 4-a). The maximum elevation difference between the highest and lowest station serves as one of our network characteristics and can 
be also used to analyze elevation patterns on a global scale. While we were considering to show this information by color coding the stations (circles), visualization is always a careful tradeoff and showing too much quickly leads to cluttered interfaces. To keep the design straightforward and to the point, we thus decided to show elevation information of stations in a separate view and connect it via linking and brushing.

Besides the elevation profiles, we further enhanced our database with hourly weather records for all cities. Weather has been found a substantial factor in bike sharing demand [10]. In addition to cross-correlation between the temperature and the network activity, which we compute for easy global comparisons, we also added an additional time series view showing this data visually. Vienna, for example, has a distinctive pattern and a particularly strong relationship between the network activity and the temperature, as shown in Figure 5. The two white cuts in April and August were caused by server issues and do not represent the actual network activity. Visualization is also good in quickly revealing such anomalies [11]. Analyzing the impact of weather conditions on bike-sharing can help to improve rebalancing operations and the planning of new systems in the future [12].

Generally, we opted for a modular design that can be easily extended with data from other sources, as well as with different views onto this data. Especially future work in urban sociology would benefit from an integration of additional context information, such as demographic developments (workplace density, gross domestic product etc).

4.2.3. Usage Scenario - Multi-City Comparisons: Conceptually, our user interface follows Shneiderman`s venerable information-seeking mantra ("overview first, zoom and filter, details on demand") [13]. In order to provide an overview, a shared global perspective on the collected data, we implemented multiple interactive visualizations of all networks, such as zoomable maps or a small multiples view (see Figure 2-a and 2-b). Due to the same scales and axes, small multiples are very efficient for giving a first quick overview and for comparison purposes [14]. The individual vector maps with bike-sharing stations as dots serve as indicators of the size, structure, and density of networks.

To illustrate the global scale of our data, we implemented another multi-coordinated view dashboard that allows users to interactively explore bike-sharing systems globally (Figure 6). Several frequency charts (or strip plots), with thin, vertical lines representing individual networks, show the distribution of networks along a set of selected dimensions, such as, population, network activity, or the reachable stations (see Section 3 for more details). Similar to the fill level analyzer, users can draw lines on top of these charts to select certain ranges of interest. Again, leveraging the linking and brushing approach, the selection is highlighted on all other dimensions, as well as in the corresponding map. Vice versa, upon filtering a specific geographical region (lasso selection, see Figure 4-b), the received values are highlighted in the frequency chart.

While operators of these systems have mostly a rather narrow and local view, our visualization enables them to explore, compare, and learn from other networks worldwide. Similarly, politicians and transportation authorities who are planning a new bike-sharing system can get interesting insights from equal-sized cities, such as the number of reachable stations in a 2 kilometer radius.

4.2.4. Usage Scenario - General Public: By bringing together distributed, heterogeneous data on a single platform we also simplify the access for general public users. While this user group is often not interested in in-depth analysis, it can benefit from a lightweight interface that leverages this rich data source. Our implemented system includes various features that illustrate how typical tasks that appeal to the general public can be supported, for example, maps showing live station fill levels or a bike-sharing route planner for all our 468 networks, shown in Figure 1-b and Figure 1-c respectively. 

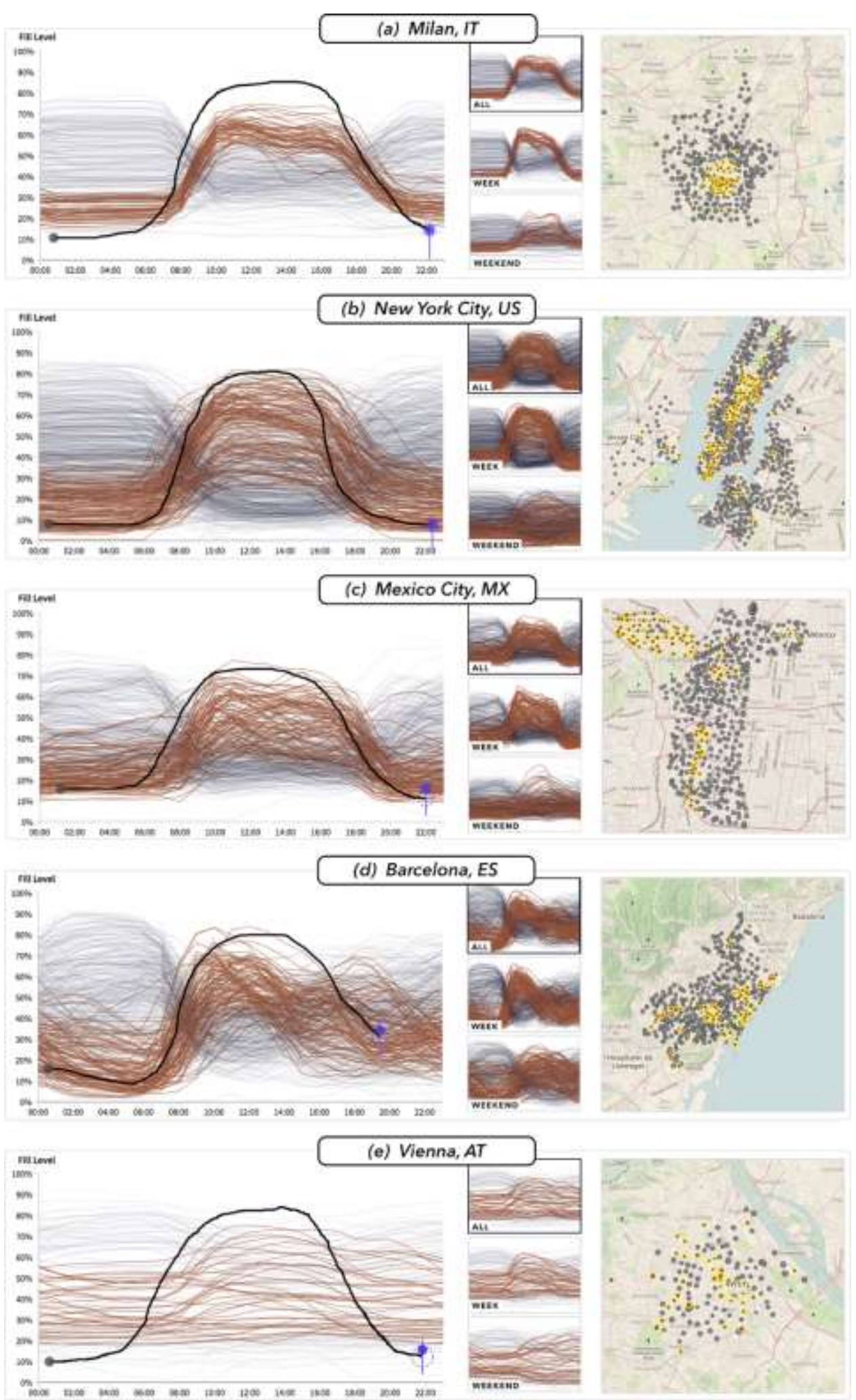

Figure 3. Station Fill Levels Visualized as Multi-Series Line Charts Serve as Indicators of Commuting Behaviors Worldwide. Small Thumbnails in the Middle Column of each View Show How Patterns Differ during the Week from those on the Weekend. Users can Click on one of the Thumbnails to show it in the Main View Enlarged. Stations can be Selected either in the Line Chart or in the Map View and are Highlighted Respectively 


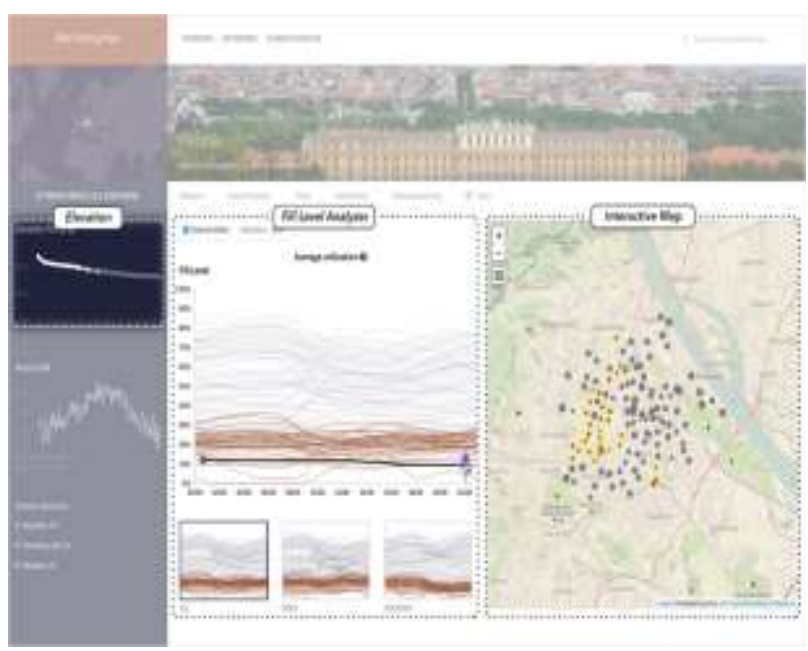

(a) Fill Level Analyzer Shows Mostly Balanced Fill Levels in Vienna but Provides Evidence of Fewer Available Bikes at Higher Altitudes

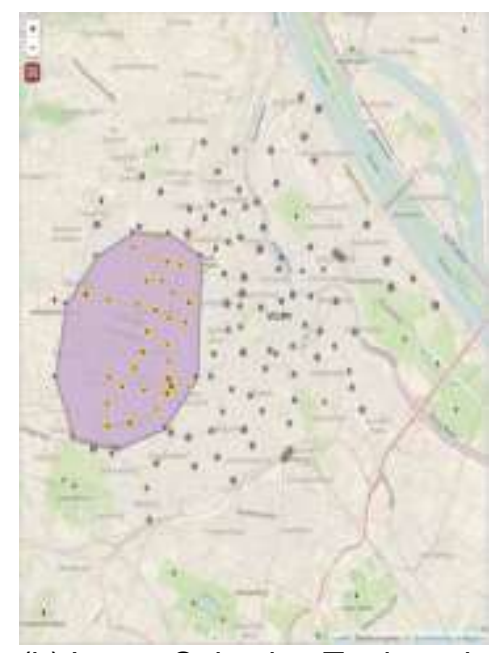

(b) Lasso Selection Tool can be used to Filter Stations based on a Geographical Region

Figure 4. Analyzing Station Fill Levels of Vienna's Bike-Sharing Network

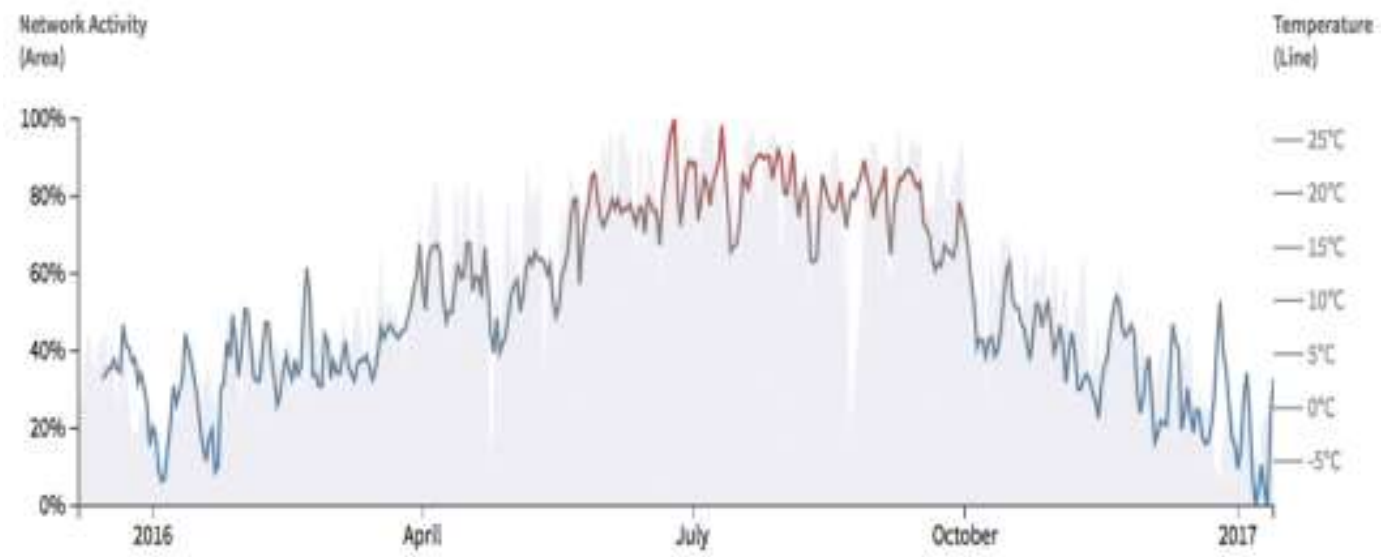

Figure 5. Time Series Showing the Network Activity (Gray Area) and the Temperature (Line) in Vienna

Within the route planner users can enter trip start- and endpoints and the system automatically finds the nearest stations and the fastest route. Additional tooltips in the interactive map show the current fill level and a historical profile for each station, which we computed by averaging across the 17 months of data that we recorded. This information can be used to roughly predict how the availability might look like at a certain point in time, similar to Google's ‘popular times' feature [15]. While similar planning tools exist for many systems [16,17], our data allows a unified approach across them.

In order to make the tool usable for a broad audience, we cannot have a steep learning curve as is the case for many expert visualization and data analysis tools. Hence, it is important that users are immediately aware of the benefits to get interested. Different design considerations and a much stronger focus on usability has been taken into account. We sought to consistently use easy to understand visual encodings, specifically as the visualization literacy of the general public is known to be low [18]. The feedback received in interactions with six persons of this user group has shown the added value, especially for those traveling or moving to new cities. 


\subsection{Implementation}

Our system is implemented as a web-based tool that runs in every modern web browser and that adapts flexible to different desktop monitors. While we have used Python extensively for data-preprocessing, the actual web-interface is primarily based on JavaScript. We use the library d3.js ${ }^{1}$ for visualizations, and leaflet.js ${ }^{2}$ for integrating interactive maps. The fill level analyzer with its interactive selection tool is also purely based on JavaScript and d3.js. The collected and aggregated data is stored in a MySQL database and partly in small CSV files that can be loaded asynchronously as needed.

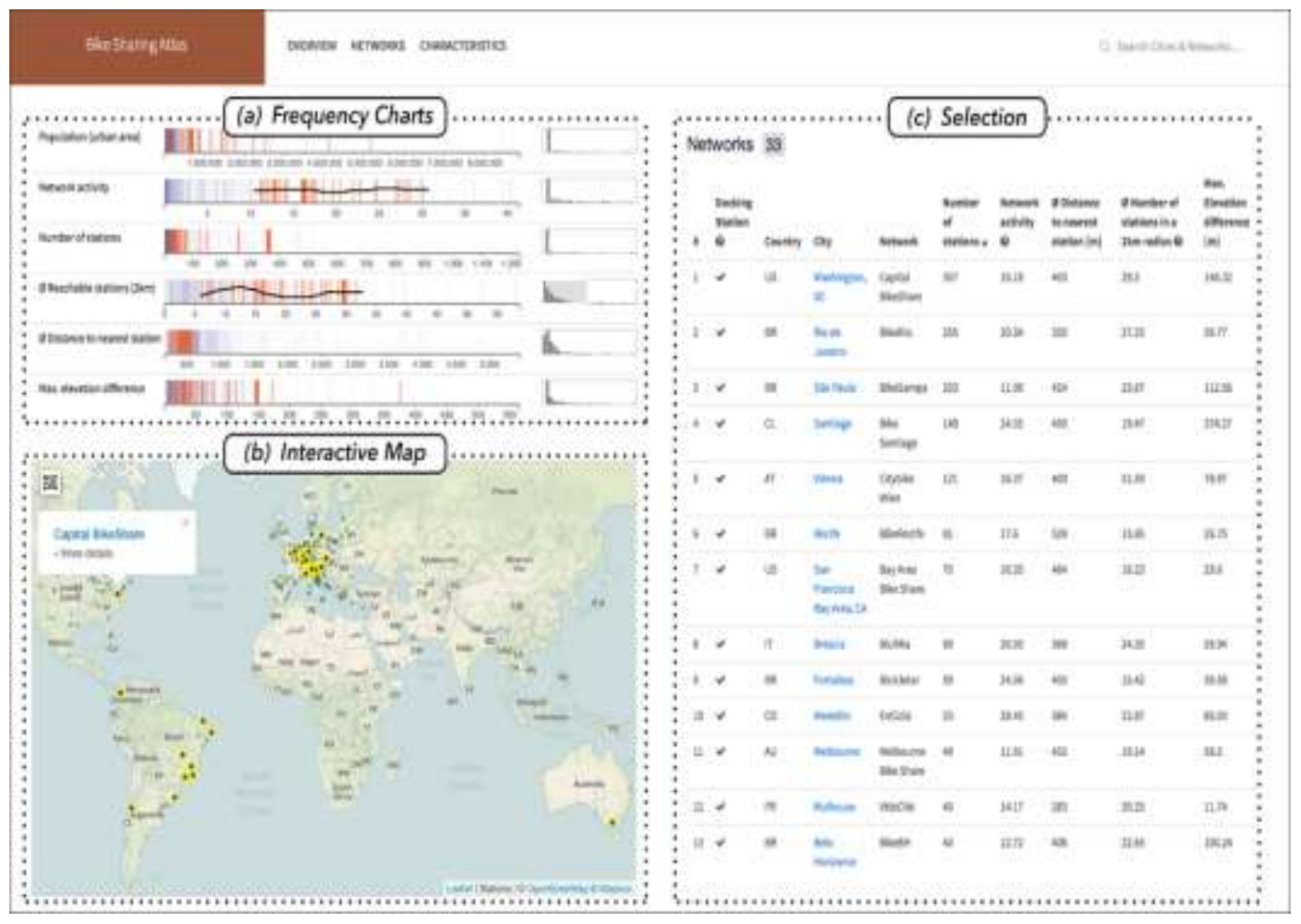

Figure 6. Frequency Charts Linked with a Map View Allow Users to Interactively Explore and Compare Network Characteristics

\section{Conclusions and Future Work}

In this work we discuss a data-driven and visual approach to understanding and leveraging smart city data. Through our iterative design process, we found evidence that such an approach can benefit different target groups. The fill level analyzer with its multicoordinated views, for instance, provides a new way to explore and communicate commuting behaviors in 45 countries. The combination with other data sources can help, for instance, urban sociology researchers to analyze effects of residential segregation. Elevation profiles support bike-sharing operators in identifying bottlenecks with stations at higher altitudes. An integrated route planner and live station fill levels offer a benefit for general public users.

While our system is primarily built around global bike-sharing data we believe that the proposed visual approach is relevant for other smart city sensors too. Observed more closely, we use the data not only to analyze cycling behavior or to build a route planner but also as a way to understand high-level city dynamics more generally. The concept of using sensors for monitoring tasks for which they were not initially designed is called

\footnotetext{
${ }^{1} \mathrm{https}: / / \mathrm{d} 3 j \mathrm{~s}$. org

${ }^{2}$ http://leafletjs.com
} 
opportunistic sensing (or citizen sensing) [35]. Massive amounts of data, produced by car sharing services, taxis, public transport systems, smart meters, or other sensors provide abstractly similar challenges and opportunities. By recording them over a long time period and by making them accessible and visually explorable it could open up new possibilities in understanding and improving urban environments.

We will continue to gather live station fill levels in a $15 \mathrm{~min}$ interval and to execute the preprocessing workflow regularly. Furthermore, it is our intention to continuously expand the platform and to integrate additional bike-sharing networks in the future.

We recorded not only the temperature but also weather conditions, humidity and wind speed for all cities. Eventually, however we decided to include only the temperature for the beginning. Thus, several questions remain unanswered such as the influence of precipitation or extreme weather conditions on the network activity. The weather records combined with bike-sharing data or other datasets open broad possibilities for detailed analyses that we plan to address in future work.

So far, we have only scratched the surface and there are many more usage scenarios that could be explored in this context. But also the ones that we have identified would benefit from being complemented, for instance, by further design studies and in-depth collaboration with specific user groups [36]. Our work of gathering and making the data available now provide the first steps towards such future endeavors. Beyond that, we also hope that our work will inspire researchers and designers of other urban data solutions.

\section{References}

[1] P. DeMaio and J. Gifford, "Will smart bikes succeed as public transportation in the United States?" Journal of Public Transportation, vol. 7, no. 2, (2004), pp. 1-15.

[2] J. Froehlich, J. Neumann and N. Oliver, "Sensing and predicting the pulse of the city through shared bicycling", in IJCAI, vol. 9, (2009), pp. 1420-1426.

[3] J. Wood, R. Beecham and J. Dykes, "Moving beyond sequential design: Reflections on a rich multichannel approach to data visualization", IEEE Transactions on Visualization and Computer Graphics, vol. 20, no. 12, (2014), pp. 2171-2180.

[4] CityBikes API, 2017, last accessed April 26, 2017. [Online]. Available: https://citybik.es

[5] C. M. de Chardon and G. Caruso, "Estimating bike-share trips using station level data," Transportation Research Part B: Methodological, vol. 78, (2015), pp. 260-279.

[6] J. Boy, L. Eveillard, F. Detienne and J.-D. Fekete, "Suggested interactivity: Seeking perceived affordances for information visualization", IEEE Transactions on Visualization and Computer Graphics, vol. 22, no. 1, (2016), pp. 639-648.

[7] O. O'Brien, J. Cheshire and M. Batty, "Mining bicycle sharing data for generating insights into sustainable transport systems", Journal of Transport Geography, vol. 34, no. 262-273, (2014).

[8] J. Heer and B. Shneiderman, "Interactive dynamics for visual analysis", Queue, vol. 10, no. 2, pp. 1-26, (2016).

[9] S. Jones, "Working 9 to 8: Spain seeks to shorten 11-hour working day", December 2016, last accessed April 26, 2017. [Online]. Available: https://www.theguardian.com/world/2016/dec/13/spain-leavesfranco-in-past- as-it-seeks-to-move-clocks-back-an-hour.

[10] M. S. Smith and G. Kauermann, "Bicycle commuting in Melbourne during the 2000s energy crisis: A semiparametric analysis of intraday volumes", Transportation Research Part B: Methodological, vol. 45, no. 10, (2011), pp. 1846-1862.

[11] S. Kandel, A. Paepcke, J. Hellerstein and J. Heer, "Wrangler: Interactive visual specification of data transformation scripts", in Proceedings of the SIGCHI Conference on Human Factors in Computing Systems. ACM, (2011), pp. 3363-3372.

[12] K. Gebhart and R. B. Noland, "The impact of weather conditions on bikeshare trips in Washington, DC", Transportation, vol. 41, no. 6, (2014), pp. 1205-1225.

[13] B. Shneiderman, "The eyes have it: A task by data type taxonomy for information visualizations", IEEE Symposium on Visual Languages, (1996), pp. 336-343.

[14] E. Tufte, "Envisioning Information", Cheshire, CT, USA: Graphics Press, (1990).

[15] Google, "Popular times", 2017, last accessed April 26, 2017. [Online]. Available: https://goo.gl/F7D0c9.

[16] Citymapper, "The ultimate transport app", 2017, last accessed April 26, 2017. [Online]. Available: https://content.citymapper.com.

[17] Mapbox, "Plan a ride with surface, directions, and turf.js", 2017, last accessed April 26, 2017. [Online]. Available: https://www.mapbox.com/bites/00094/. 
[18] J. Boy, R. A. Rensink, E. Bertini and J.-D. Fekete, “A principled way of assessing visualization literacy”, IEEE Transactions on Visualization and Computer Graphics, vol. 20, no. 12, (2014), pp. 1963-1972.

[19] J. C. García-Palomares, J. Gutiérrez and M. Latorre, "Optimizing the location of stations in bike-sharing programs: a GIS approach", Applied Geography, vol. 35, no. 1, (2012), pp. 235-246.

[20] C. Kloimüllner, P. Papazek, B. Hu and G. R. Raidl, "Balancing bicycle sharing systems: an approach for the dynamic case", in European Conference on Evolutionary Computation in Combinatorial Optimization, (2014), pp. 73-84.

[21] T. Munzner, "Visualization Analysis and Design", CRC Press, (2014)

[22] G. Andrienko, N. Andrienko, P. Bak, D. Keim and S. Wrobel, "Visual analytics of movement", Springer Science \& Business Media, (2013).

[23] R. Beecham, J. Wood and A. Bowerman, "Studying commuting behaviours using collaborative visual analytics", Computers, Environment and Urban Systems, vol. 47, (2014) September, pp. 5-15.

[24] G. N. Oliveira, J. L. Sotomayor, R. P. Torchelsen, C. T. Silva and J. L. Comba, "Visual analysis of bikesharing systems", Computers \& Graphics, vol. 60, (2016), pp. 119-129.

[25] Bostonography, "Hubway trip explorer", 2017, last accessed April 26, 2017. [Online]. Available: http://bostonography.com/hubwaymap/.

[26] T. von Landesberger, F. Brodkorb, P. Roskosch, N. Andrienko, G. Andrienko and A. Kerren, "Mobilitygraphs: Visual analysis of mass mobility dynamics via spatio-temporal graphs and clustering", IEEE Transactions on Visualization and Computer Graphics, vol. 22, no. 1, (2016), pp. 11-20.

[27] L. Yu, W. Wu, L. Xiaohui, L. Guangxia, W. Siong Ng, S.-K. Ng, Z. Huang, A. Arunan and H. M. Watt, "iVizTRANS: Interactive visual learning for home and work place detection from massive public transportation data", in IEEE Conference on Visual Analytics Science and Technology (VAST), (2015), pp. 49-56.

[28] N. Ferreira, J. Poco, H. T. Vo, J. Freire and C. T. Silva, "Visual exploration of big spatio-temporal urban data: A study of New York City taxi trips", IEEE Transactions on Visualization and Computer Graphics, vol. 19, no. 12, (2013), pp. 2149-2158.

[29] F. Miranda, H. Doraiswamy, M. Lage, K. Zhao, B. Gonçalves, L. Wilson, M. Hsieh and C. T. Silva, "Urban pulse: Capturing the rhythm of cities", IEEE Transactions on Visualization and Computer Graphics, vol. 23, no. 1, (2017), pp. 791-800.

[30] M. Z. Austwick, O. O'Brien, E. Strano, and M. Viana, "The structure of spatial networks and communities in bicycle sharing systems", PLoS ONE, vol. 8, no. 9, (2013), p. e74685.

[31] A. Bargar, A. Gupta, S. Gupta and D. Ma, "Interactive visual analytics for multi-city bikeshare data analysis", in 3rd International SIGKDD Workshop on Urban Computing (UrbComp), (2014).

[32] T. Nagel, C. Pietsch and M. Dörk, "Staged analysis: From evocative to comparative visualizations of urban mobility", Proceedings of the IEEE VIS Arts Program, VISAP, (2016), pp. 23-30.

[33] O. O'Brien, "Bike share map", September 2017, last accessed April 26, 2017. [Online]. Available: http://bikes.oobrien.com.

[34] R. Meddin and P. DeMaio, "The bike-sharing world map", September 2017, last accessed April 26, 2017. [Online]. Available: http://bikesharingmap.com.

[35] D. Havlik, S. Schade, Z. A. Sabeur, P. Mazzetti, K. Watson, A. J. Berre and J. L. Mon, "From sensor to observation web with environmental enablers in the future internet", Sensors, vol. 11, no. 4, (2011), pp. 3874-3907.

[36] M. Sedlmair, M. Meyer and T. Munzner, "Design study methodology: Reflections from the trenches and the stacks", IEEE Transactions on Visualization and Computer Graphics, vol. 18, no. 12, (2012), pp. 24312440 .

[37] C. Weaver, "Building highly-coordinated visualizations in improvise", in IEEE Symposium on Information Visualization, (2004), pp. 159-166.

[38] B. Shneiderman, "Direct manipulation: A step beyond programming languages", IEEE Computer, vol. 16, no. $8,(\mathbf{1 9 8 3})$, pp. $57-69$.

[39] C. Ware, Information visualization: perception for design. Elsevier, (2012).

\section{Authors}

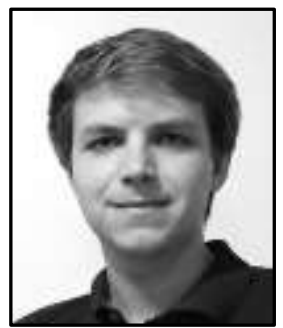

Michael Oppermann is currently in the $\mathrm{PhD}$ program of the Department of Computer Science at the University of British Columbia. He received his Dipl.-Ing. degree (MSc) in Business Informatics from the University of Vienna. His research interests include information visualization, human-computer interaction and data-driven journalism. 


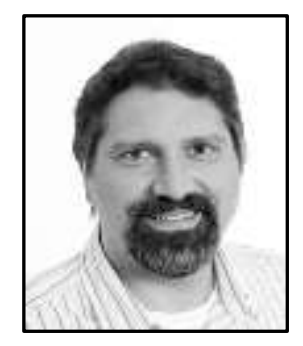

Torsten Möller is a professor at the University of Vienna, Austria, since 2013. Between 1999 and 2012 he served as a Computing Science faculty member at Simon Fraser University, Canada. He received his $\mathrm{PhD}$ in Computer and Information Science from Ohio State University in 1999 and a Vordiplom (BSc) in mathematical computer science from Humboldt University of Berlin, Germany. He is a senior member of IEEE and ACM, and a member of Eurographics. His research interests include algorithms and tools for analyzing and displaying data with principles rooted in computer graphics, human-computer interaction, image processing, machine learning and visualization.

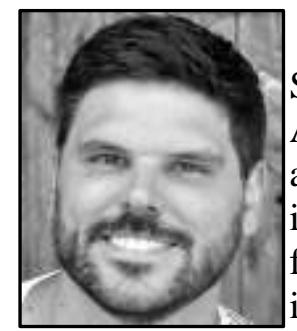

Michael Sedlmair received his $\mathrm{PhD}$ degree in Computer Science from the Ludwig Maximilians University of Munich. After a postdoc at the University of British Columbia, he is now an assistant professor at the University of Vienna. His research interests include visualization design and evaluation, human factors in visualization and data analysis, and human-computer interaction. 